\title{
A SAÚDE DO ADOLESCENTE IMIGRANTE: UMA REVISÃO SISTEMÁTICA
}

\author{
IMMIGRANT TEEN HEALTH: A SYSTEMATIC REVIEW
}

Caroline Terrazas ${ }^{1}$, Maria Sylvia de Souza Vitalle²

\section{RESUMO}

Questões da adolescência costumam ser potencializadas em adolescentes imigrantes. Assim, o objetivo desta revisão é identificar o que, atualmente, se discute sobre a saúde destes adolescentes. Foi realizada uma revisão sistemática na base de dados PubMed e foram localizados 96 artigos. A seguir, foram feitas a seleção inicial, leitura e identificação dos trabalhos que tratavam exclusivamente sobre saúde e adolescente imigrante. Dos três trabalhos selecionados ao final deste processo, um aponta para o stress relacionado à adaptação cultural de jovens imigrantes, incluindo quadros de depressão e ansiedade; o segundo artigo refere-se ao consumo de álcool, tabaco e maconha por adolescentes com e sem antecedentes de imigração; e o terceiro aponta para o trauma e saúde mental de adolescentes durante os períodos da pré-migração, migração e pós-migração. Apesar do intenso movimento migratório e do quanto isto afeta a saúde desta população, os trabalhos que abordam a saúde dos adolescentes são escassos e oferecem poucas informações relevantes sobre esta questão.

DESCRITORES: Imigração. Saúde do Adolescente. Adolescente. Emigração. Comportamento do Adolescente

\section{ABSTRACT}

Adolescent issues are often raised in immigrant adolescents. Thus, the aim of this review is to identify what is currently being discussed about the health of these adolescents. A systematic review of the PubMed database was performed and 96 articles were found. Subsequently, the initial selection, reading and identification of papers that dealt exclusively with health and adolescent immigrants were made. Of the three papers selected at the end of this process, one points to stress related to cultural adaptation of young immigrants, including depression and anxiety; The second article refers to the consumption of alcohol, tobacco and marijuana by adolescents with and without previous immigration. and the third points to the trauma and mental health of adolescents during the pre-migration, migration and post-migration periods. Despite the intense migratory movement and how much this affects the health of this population, studies addressing adolescent health are scarce and offer little relevant information on this issue. KEYWORDS: Immigration. Adolescent Health. Adolescent. Emigration. Adolescent Behavior

1 Enfermeira. Doutoranda do Programa de Pós-graduação ESIA-UNIFESP. Guarulhos, SP, Brasil. E-mail: carotera@gmail.com

2 Professor Permanente do ESIA-UNIFESP. Prof. ${ }^{a}$ Adjunto Doutor e Chefe do Setor de Medicina do Adolescente - Escola Paulista de Medicina/UNIFESP, São Paulo, SP, Brasil. E-mail: sylviavitalle@gmail.com 


\section{INTRODUÇÃO}

Os movimentos migratórios foram a base de formação de muitas civilizações multiétnicas pelo mundo. Atualmente, os padrões de migração mundial apresentam, como característica, a globalização, que envolve um maior número de países remetentes e destinatários (GIDDENS, 2008) e contribui, também, para uma distribuição desigual de oportunidades socioeconômicas, resultando em um grande número de deslocamentos para outros países. Dados da International Organization for Migration (2010) mencionam que há 214 milhões de migrantes no mundo, isto é, $3,2 \%$ da população mundial. A maioria das migrações é realizada por motivos econômicos, com alto impacto da imigração ilegal. Define-se como imigração a mudança de pessoas ou populações de um país para outro, de forma temporária ou permanente. Os fluxos migratórios internacionais caracterizam-se por desejos e aspirações de mudanças de vida fora de seu local de origem (DIAS, 2013). A possibilidade de uma mudança de país representa uma fonte de enriquecimento social, cultural e econômico. Mas é, também, uma fonte de problemas e conflitos sociais.

A mobilidade internacional tem fundamental importância na atualidade, em função do aumento intenso e diversificado do fluxo e circulação humana e de deslocamentos forçados ou voluntários. A complexidade dos deslocamentos atuais coloca em evidência a necessidade de se construírem modelos explicativos abrangentes e interdisciplinares, que busquem alcançar a multifatorialidade na explicação dos fenômenos relacionados às migrações e à saúde, e que se constituam na interface dos diferentes campos do saber (GOLDBERG, MARTIN E SILVEIRA, 2015). Ao longo do tempo, observa-se que os imigrantes são grupos particularmente vulneráveis a uma diversidade de fatores que determinam as suas condições de saúde. No plano internacional, diversas questões têm sido levantadas com relação às especificidades da condição dos imigrantes e às desvantagens cumulativas sofridas por essa população no acesso à saúde. Há uma importância crescente do tema saúde na agenda da União Europeia, seja na defesa da igualdade de acesso aos serviços prestados, seja na melhoria da sua qualidade. A relação entre saúde e imigração ganha importância nas preocupações dos responsáveis políticos e dos investigadores que estudam a inserção e saúde dos imigrantes nos países de acolhimento (RAMOS, 2016).
Os adolescentes imigrantes são o foco deste estudo pois, além de lidarem com todos os fatores de risco descritos anteriormente, também enfrentam os desafios normativos deste período de desenvolvimento - incluindo a formação da identidade, a pressão dos colegas, transição para o ensino médio, a construção individual da identidade, mudança no papel dos adultos significativos, a relevância dos parentes e familiares e a adaptação às mudanças da puberdade (STEINBERG et al, 2006; RAMOS, 2016). Os jovens imigrantes, como todos os outros, necessitam ajustar-se com sucesso às tarefas típicas da sua fase de vida, assim como às funções exigidas pelos diferentes contextos. Contudo, no caso dos imigrantes, a situação se agrava na medida em que vivem entre duas realidades culturais distintas (HERNANDEZ, DENTON, MACARTNEY, 2008). Trata-se de um processo estressante associado, no caso de adolescentes, aos desafios relacionados com a adaptação ao contexto escolar, ao grupo de amigos, ao ambiente cultural e social, e, muitas vezes, a um novo idioma. Além disso, nesta fase, os adolescentes imigrantes podem estar mais vulneráveis à exposição a fatores de risco, sendo que o processo de integração pode resultar em consequências graves e negativas, de longa duração.

Observa-se que algumas das dificuldades encontradas durante a adolescência podem ser agravadas no que se refere à saúde do adolescente imigrante, uma vez que esses, em especial, representam um grupo vulnerável. Desse modo, há que se pensar na compreensão das relações entre imigração, saúde e adolescentes. No Brasil, há pouca produção científica referente à saúde do adolescente imigrante. Por isso, o objetivo desse estudo foi identificar o que se discute, atualmente, sobre a saúde do adolescente imigrante, por meio de uma revisão sistemática de 5 anos.

\section{MÉTODO}

Este estudo é uma revisão sistemática da literatura científica, sem metanálise, sobre o tema saúde do adolescente imigrante. A pesquisa foi realizada de acordo com as recomendações metodológicas PRISMA (Preferred Reporting Items for Systematic Reviews) para estudos de revisão sistemática (MOHER et al, 2009). Definimos a seguinte questão norteadora: quais são os estudos que falam da saúde do adolescente imigrantes e problemas de saúde dessa população? 
Para a construção dessa pergunta e dos critérios de elegibilidade, utilizamos o acrônimo PICO: P (patient) - adolescentes imigrantes; I (intervention) - não se aplica ao estudo; C (comparation group) - não se aplica ao estudo; e O (outcome) - saúde do adolescente imigrante e agravos à saúde de adolescentes imigrantes (BRASIL, 2012). Os artigos foram pesquisados na base de dados eletrônica PubMed nos idiomas português e inglês, no período de 2014 a 2019, a partir dos descritores "emigração", "imigração", "saúde do adolescente", "adolescente", "emigration", "immigration", "adolescent health" e "adolescent". Foram considerados os seguintes critérios de inclusão para seleção dos artigos: descritores pesquisados constando no título ou no resumo; resultados constando aspectos relacionados à saúde do adolescente imigrante suicida e/ou tentativa de suicídio em universitários; artigos publicados, na íntegra ou resumo, no período de 1988 a 2018, nos idiomas português/ inglês. Como critério de exclusão, foi considerado artigo que, embora falasse sobre a saúde ou doenças de imigrantes, não incluísse os adolescentes.

Inicialmente, foram identificados 96 trabalhos. Após a leitura do título, foram excluídos 71 , que não eram pertinentes ao tema e à população, resultando em 25 artigos. Desses restantes, após avaliação do título e resumo, 14 foram selecionados. A amostra resultante foi de 3 artigos que tratavam exclusivamente sobre o tema, como apresentado, abaixo, na Figura 1:

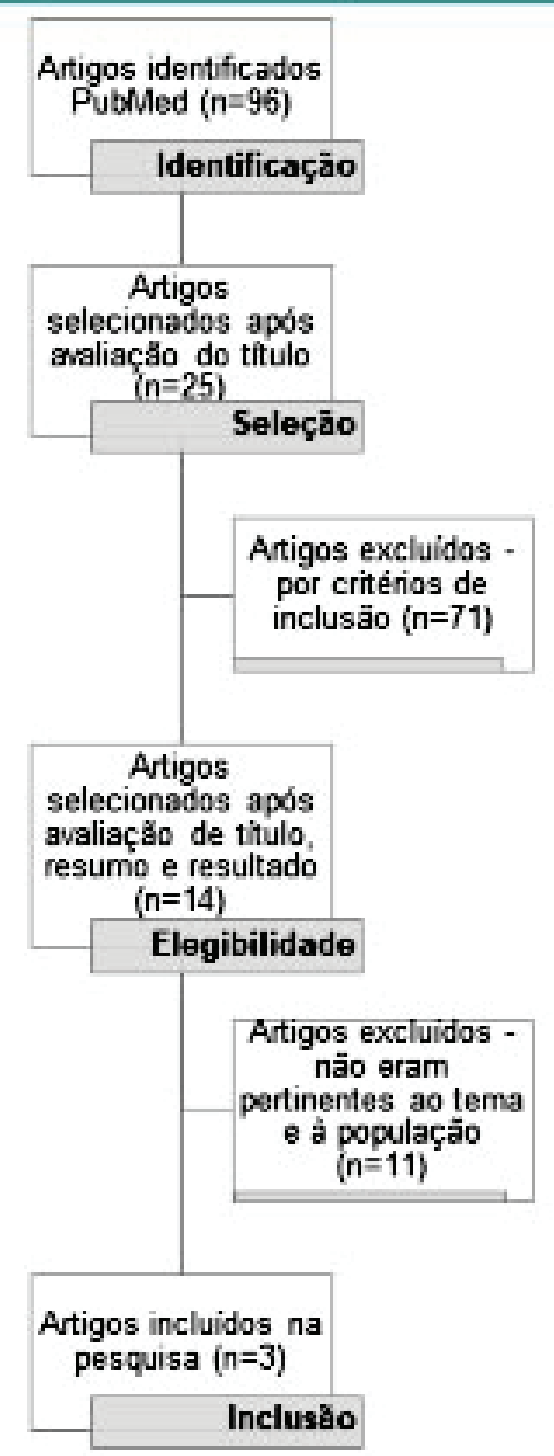

Figura 1 - Método de identificação e seleção dos artigos 


\section{RESULTADOS}

Dos três artigos selecionados, o primeiro aponta para o stress relacionado à adaptação cultural de jovens imigrantes, incluindo quadros de depressão e ansiedade; o segundo refere-se ao consumo de álcool, tabaco e maconha por adolescentes com e sem antecedentes de imigração; e o terceiro artigo aponta para o trauma e saúde mental de adolescentes durante os períodos da pré-migração, migração e pós-migração, conforme apresentado, abaixo, no Quadro 1.

Quadro 1 - Resultados - São Paulo- 2019

\begin{tabular}{|c|c|c|c|c|c|}
\hline Autores e Ano & Título & Tipo de estudo & Local & Amostra & Resultados \\
\hline $\begin{array}{l}\text { Cleary, S.D.; } \\
\text { Snead, R.; } \\
\text { Chavez, D.D.; } \\
\text { Rivera, I.; } \\
\text { Edberg, M.C. } \\
2018\end{array}$ & $\begin{array}{l}\text { Immigrant Trauma and } \\
\text { Mental Health Outcomes } \\
\text { Among Latino Youth: } \\
\text { (Trauma imigrante e } \\
\text { resultados de saúde } \\
\text { mental entre jovens } \\
\text { latinos) }\end{array}$ & $\begin{array}{l}\text { Análise } \\
\text { quantitativa }\end{array}$ & Estados Unidos & $\begin{array}{l}\text { Jovens sul } \\
\text { americanos, faixa } \\
\text { etária de } 12 \text { a } 17 \\
\text { anos, que vivem } \\
\text { nos USA há menos } \\
\text { de } 3 \text { anos. } \\
\text { Total: } 104 \\
\text { adolescentes }\end{array}$ & $\begin{array}{l}\text { Destes, } 59 \% \\
\text { relataram que o } \\
\text { evento ocorreu em } \\
\text { seu país de origem, } \\
20 \% \text { relataram ter } \\
\text { vivenciado o evento } \\
\text { durante a migração } \\
\text { e } 18 \% \text { relataram um } \\
\text { evento desde sua } \\
\text { chegada aos EUA. }\end{array}$ \\
\hline $\begin{array}{l}\text { Donath, C.; } \\
\text { Baier, D.; } \\
\text { Graessel, E.; } \\
\text { Hillemacher, T } \\
2016\end{array}$ & $\begin{array}{l}\text { Substance consumption } \\
\text { in adolescents with } \\
\text { and without an } \\
\text { immigration background: } \\
\text { a representative } \\
\text { study_What part of an } \\
\text { immigration background } \\
\text { is protective against binge } \\
\text { drinking? } \\
\text { (Consumo de substâncias } \\
\text { em adolescentes com } \\
\text { e sem antecedentes de } \\
\text { imigração: um estudo } \\
\text { representativo - Que parte } \\
\text { de um contexto migratório } \\
\text { protege contra o consumo } \\
\text { excessivo de álcool?) }\end{array}$ & $\begin{array}{l}\text { Análise } \\
\text { quantitativa }\end{array}$ & Alemanha & $\begin{array}{l}\text { Adolescentes, faixa } \\
\text { etária de } 12 \text { a } 15 \\
\text { anos } \\
9.512 \text { alunos, } \\
\text { desses } 1763 \text { com } \\
\text { antecedentes } \\
\text { migratórios }\end{array}$ & $\begin{array}{l}\text { Distinções devem } \\
\text { ser feitas em relação } \\
\text { à substância, ao } \\
\text { país de origem do } \\
\text { adolescente. }\end{array}$ \\
\hline $\begin{array}{l}\text { Frounfelker, } \\
\text { R. L.; Assefa, } \\
\text { M.T.; Smith, E.; } \\
\text { Hussein, A.; } \\
\text { Betancourt, T } \\
2018\end{array}$ & $\begin{array}{l}\text { "We would never } \\
\text { forget who we are": } \\
\text { resettlement, cultural } \\
\text { negotiation, and family } \\
\text { relationships among } \\
\text { Somali Bantu refugees. } \\
\text { (“Jamais esqueceremos } \\
\text { quem somos": } \\
\text { reassentamento, } \\
\text { negociação cultural e } \\
\text { relações familiares entre } \\
\text { refugiados somalis bantu) }\end{array}$ & $\begin{array}{l}\text { Análise } \\
\text { qualitativa }\end{array}$ & Estados Unidos & $\begin{array}{l}\text { Adolescentes faixa } \\
\text { etária: } 10 \text { a } 17 \text { anos } \\
\text { Total: } 47\end{array}$ & $\begin{array}{l}\text { Os jovens somalis } \\
\text { bantos enfrentaram } \\
\text { discriminação por } \\
\text { causa de suas } \\
\text { identidades como } \\
\text { africanos, somalis } \\
\text { e muçulmanos e } \\
\text { bullying no ambiente } \\
\text { escolar. }\end{array}$ \\
\hline
\end{tabular}




\section{DISCUSSÃO}

Estudos têm mostrado que adolescentes oriundos de grupos étnico-culturais minoritários, pela peculiaridade das pressões, estão sujeitos a aculturação, discriminação, racismo, desenraizamento, intolerância, preconceito e podem demonstrar alterações conflitantes na identidade social e autoconceito, assim como uma tendência para evidenciar prevalência de condutas problemáticas (GASPAR, 2006; MOTTI-STEFANIDI, 2008; GASPAR, MATOS, 2009; LEMOS, NUNES, GUIMARÃES, 2011). Além disso, estão igualmente mais propensos a enfrentar a discriminação e vitimização (HUYNH, FULIGNI, 2010).

Neste sentido, durante a adolescência, são vários contextos que poderão contribuir de forma propícia ou prejudicial para o crescimento e desenvolvimento do jovem, sendo que os desajustamentos, ao nível destes contextos, poderão resultar numa condição emocional instável e/ou psicologicamente perturbada. Assim, se origina um aumento significativo da tendência para estes jovens desenvolverem comportamentos de risco. Quando a sociedade ou comunidade que o rodeia não lhe providencia a confiança e a estabilidade necessárias para que o desenvolvimento do adolescente decorra dentro da normalidade (SILVA, MURTA, 2009), este opta, muitas vezes, pelo caminho da delinquência e consumo de substâncias (MATOS et al, 2003; MESCH, TURJEMAN, FICHMAN, 2008). As principais dificuldades que dizem a respeito da saúde do adolescente imigrante estão diretamente relacionadas à saúde mental e ao sofrimento psíquico que esses jovens passam durante todo o processo migratório. Os resultados dessa revisão sistemática revelaram que boa parte dos adolescentes imigrantes apresentaram algum risco à saúde.

De acordo com os jovens sul americanos que residem nos Estados Unidos há menos de três anos, cuja faixa etária é de 12 a 17 anos, 59\% relataram que sofreram algum trauma no seu país de origem, $20 \%$ disseram terem vivenciado durante a imigração e $18 \%$ relataram que o trauma foi sofrido após a chegada ao país de destino (CLEARY et al, 2018). É importante esclarecer que o processo migratório é composto por três etapas, que envolvem o período pré-imigratório, trans e pós imigratório. O período pré-imigratório é aquele que antecede a imigração, o trans é o momento da mudança e o pós é a chegada e inserção do imigrante no país de destino. Sendo assim, no estudo com adolescentes sul-americanos, realizado nos Estados Unidos, a decisão de imigrar pode estar relacionada a traumas vividos nos seus países de origem e, dessa forma, a imigração foi possibilidade de mudança de vida, fora do seu local de origem.

Sobre o consumo de substâncias psicoativas, como o álcool, o tabaco e a maconha, por adolescentes alemães cuja faixa etária é de, aproximadamente, 15 anos, com e sem antecedentes de imigração, foi identificado que os jovens com antecedentes imigratórios tiveram um maior consumo de tabaco e maconha (DONATH et al, 2016).

Com relação ao sofrimento causado em adolescentes de grupos étnico-culturais minoritários residentes dos Estados Unidos, jovens entre 10 a 17 anos da etnia somalis bantos relataram, em um estudo qualitativo, terem enfrentado discriminação e bullying por conta da identidade de origem africana e por serem muçulmanos (FROUNFELKER et al, 2017).

\section{CONCLUSÃO}

Os resultados apontam para a necessidade maior de estudos que envolvam saúde, adolescentes e imigração. Apesar do intenso movimento migratório e do quanto esse processo pode afetar a saúde da população, trabaIhos que abordam temas relacionados à saúde do adolescente imigrante, em especial, são escassos, oferecendo poucas informações relevantes sobre esta questão.

Esperamos que as informações levantadas nesse estudo possam contribuir para o aprimoramento e aprofundamento de estudos e ações que atendam às necessidades e especificidades de jovens imigrantes, como também para a formulação de estratégias de cuidado aos adolescentes que estão no processo migratório.

Recomendamos novas pesquisas envolvendo adolescentes imigrantes, verificando não somente aspectos relacionados à saúde, mas também outros aspectos que envolvam a multifatorialidade dos processos migratórios atuais. 


\section{REFERÊNCIAS}

BRASIL. Ministério da Saúde. Secretaria de Ciência, Tecnologia e Insumos Estratégicos. Departamento de Ciência e Tecnologia. Diretrizes metodológicas: elaboração de revisão sistemática e metanálise de ensaios clínicos randomizados. Brasília: Editora do Ministério da Saúde, 2012.

CLEARY, Sean D. et al. Immigrant Trauma and Mental Health Outcomes Among Latino Youth. J Immigr Minor Health, v. 20, n. 5, p. 1053-1059. Oct 2018.

DIAS, Luciana de Oliveira. Goianos(as) no Mundo: um diagnóstico dos processos migratórios internacionais. In: ENCONTRO ANUAL DAANPOCS, 37, 2013 Sep 2327, Águas de Lindóia. Anais do... ST31 Migrações, Trabalho e Capitais, Águas de Lindóia, 2013.

DONATH, Carolin. et al. Substance consumption in adolescents with and without an immigration background: a representative study-What part of an immigration background is protective against binge drinking? BMC Public Health, v. 16, n. 1, p. 1157, Nov. 2016.

FROUNFELKER, Rochelle L. et al. "We would never forget who we are": resettlement, cultural negotiation, and family relationships among Somali Bantu refugees. Eur Child Adolesc Psychiatry, v. 26, n. 11, p. 1387-1400, Nov. 2017.

GASPAR, Tania. et al. Qualidade de vida e bem-estar em crianças e adolescentes. Rev. Bras. Ter. Cogn., Rio de Janeiro, v. 2, n. 2, p. 47-60, dez. 2006.

GASPAR, Tania; MATOS, Margarida Gaspar de. Adolescents lifestyles, ethnicity and socioeconomic status in Portugal. Cognition, Brain, Behavior, v. 13, n. 1, p. 49-57, 2009.

GIDDENS, Anthony. Sociologia. 6. ed. Penso, 2008.

GOLDBERG, Alejandro; MARTIN, Denise; SILVEIRA, Cássio. Editorial. Interface - Comunicação, Saúde, Informação. Botucatu, SP, v. 19, n. 53, p. 229-232, 2015.
GUIMARÃES, Sofia; LEMOS, Ida; NUNES, Cristina. Social competence and academic achievement in immigrant adolescents in Portugal. Análisis y Modificación de Conducta. v. 38, n. 157-158, p. 27-38, 2012.

HERNANDEZ, Donald J.; DENTON, Nancy A.; MACARTNEY, Suzanne. Children in immigrant families: looking to america's future. Social policy report. v. 22 , n. 3, p. 3-23, 2008.

HUYNH, Virginia W.; FULIGNI, Andrew J. Discrimination Hurts: The Academic, Psychological, and Physical Well Being of adolescents. Journal of Research on Adolescence. v. 20, n. 4, p. 916-941, 2010.

INTERNATIONAL ORGANIZATION FOR MIGRATION. World migration report 2010 The future of migration: building capacities for change. Geneva: IOM, 2010.

LEMOS, Ida; NUNES, Cristina; GUIMARÃES, Sofia. Stressful life events and perceived well-being in immigrant adolescents form Portugal. International Journal of Development and Educational Psychology. v. 2, n. 1, p. 371-380, 2011.

MATOS, Margarida Gaspar. et al. Adolescentes e o Tabaco: rapazes e raparigas. Lisboa: FMH, CPT e Ministério da Saúde, 2003.

MESCH, Gustavo S.; TURJEMAN, Hagit; FICHMAN, Gideon. Perceived discrimination and the well-being of immigrant adolescents. Journal Youth Adolescence. v. 37, n. 5, p. 592-604, May 2008.

MOHER, David. et al. Preferred Reporting Items for Systematic Reviews and Meta-Analyses: the PRISMA Statement. Annals of Internal Medicine. v. 151, n. 4, p. 264-269, 2009.

MOTTI-STEFANIDI, Frosso A. et al. Immigration as a risk factor for adolescent adaptation in Greek urban schools. European Journal of Developmental Psychology. v. 5, n. 2, p. 235-261, 2008. 
RAMOS, Maria Natália Pereira. Comunicação em Saúde e Interculturalidade - Perspectivas Teóricas, Metodológicas e Práticas. Revista Eletrônica de Comunicação, Informação e Inovação em Saúde. [S.I.], v. 6, n. 4, mar. 2016.

SILVA, Mariana de Paula; MURTA, Sheila Giardini. Treinamento de habilidades sociais para adolescentes: Uma experiência no programa de atenção integral a família (PAIF). Psicol. Reflex. Crit., Porto Alegre. v. 22, n. 1, p. 136-143, 2009.
STEINBERG, Laurence. et al. Psychopathology in adolescence: Integrating affective neuroscience with the study of context: Developmental neuroscience. Developmental psychopathology: Developmental neuroscience. 2. ed., v. 2, p. 710-741. New York, 2006.

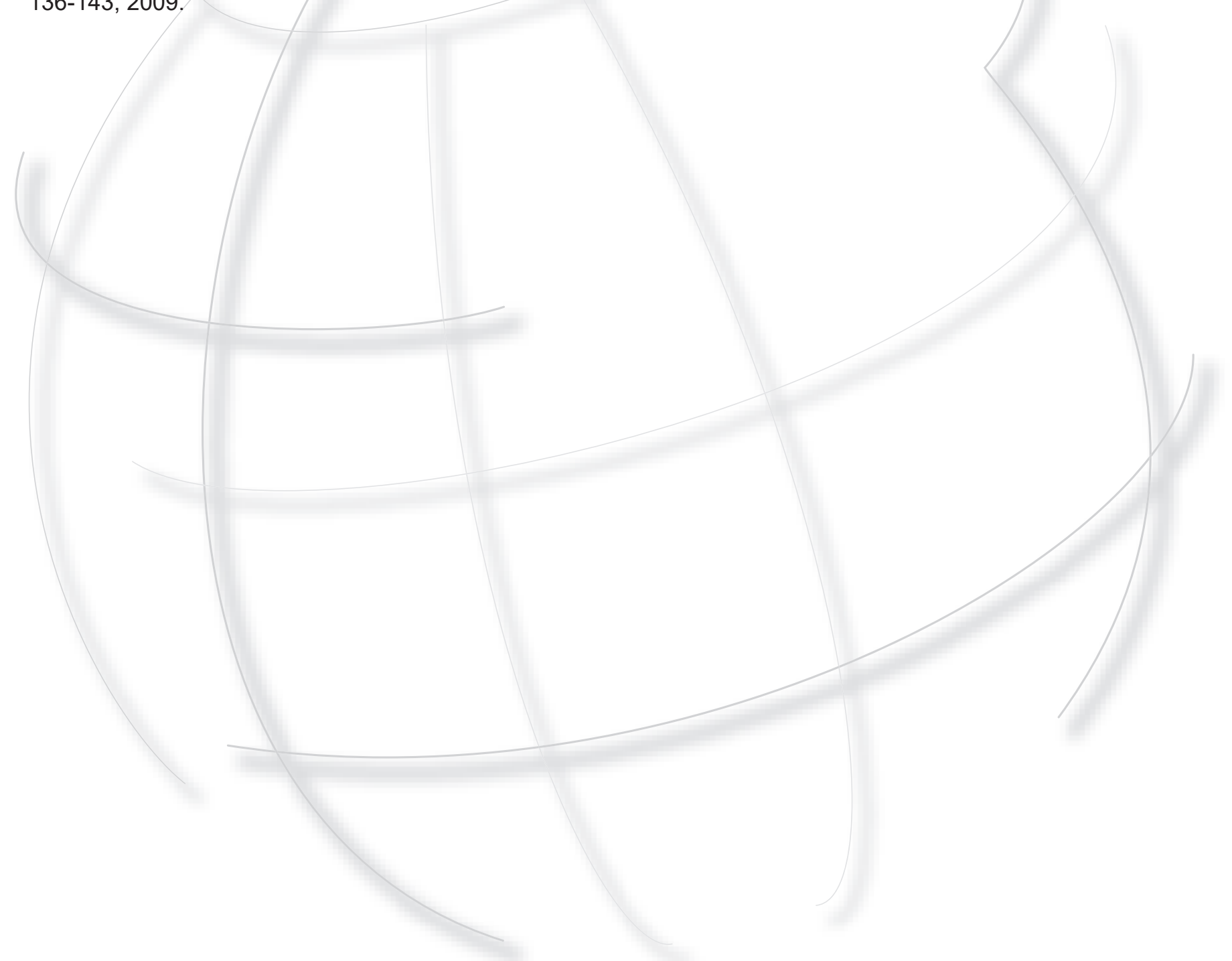

